\title{
Exact Periodic Wave Solutions to the Nizhnik-Novikov-Veselov Equation*
}

\author{
Y.-Z. PENG ${ }^{\dagger}$ \\ Department of Mathematics, Huazhong University of Science \\ and Technology, Wuhan 430074, P.R. China \\ Department of Mathematics and Physics, Henan University \\ of Science and Technology, Luoyang 471003, P.R. China
}

(Received November 3, 2003; revised version April 8, 2004)

Exact periodic wave solutions to the Nizhnik-Novikov-Veselov equation are obtained by means of the modified mapping method. Limit cases are studied and new solitary wave solutions and trigonometric periodic wave solutions are found.

PACS numbers: 02.30.Jr

\section{Introduction}

There are many methods for finding special solutions to a nonlinear partial differential equation (PDE). Some of the most important methods are the inverse scattering transformation (IST), bilinear method, symmetry reductions, Bäcklund and Darboux transformations, and so on. Recently, the exact periodic wave solutions in terms of the Jacobi elliptic functions for nonlinear PDEs attract considerable interest [1-9]. The Jacobi elliptic functions $\operatorname{sn} \xi=\operatorname{sn}(\xi \mid m), \operatorname{cn} \xi=\operatorname{cn}(\xi \mid m)$, and $\operatorname{dn} \xi=\operatorname{dn}(\xi \mid m)$, where $m(0<m<1)$ is the modulus of the elliptic function, are double periodic and possess properties of trigonometric functions, namely,

$$
\begin{aligned}
& \operatorname{sn}^{2} \xi+\mathrm{cn}^{2} \xi=1, \quad \operatorname{dn}^{2} \xi+m^{2} \operatorname{sn}^{2} \xi=1, \quad(\operatorname{sn} \xi)^{\prime}=\operatorname{cn} \xi \operatorname{dn} \xi, \\
& (\operatorname{cn} \xi)^{\prime}=-\operatorname{sn} \xi \operatorname{dn} \xi, \quad(\operatorname{dn} \xi)^{\prime}=-m^{2} \operatorname{sn} \xi \operatorname{cn} \xi .
\end{aligned}
$$

*This work has been supported by the Postdoctoral Science Foundation of China.

†e-mail: yanzepeng@163.com 
When $m \rightarrow 0$, the Jacobi elliptic functions degenerate to the trigonometric functions, i.e.,

$$
\operatorname{sn} \xi \rightarrow \sin \xi, \quad \operatorname{cn} \xi \rightarrow \cos \xi, \quad \operatorname{dn} \xi \rightarrow 1 .
$$

When $m \rightarrow 1$, the Jacobi elliptic functions degenerate to the hyperbolic functions, i.e.

$$
\operatorname{sn} \xi \rightarrow \tanh \xi, \quad \operatorname{cn} \xi \rightarrow \operatorname{sech} \xi, \quad \operatorname{dn} \xi \rightarrow \operatorname{sech} \xi .
$$

Detailed explanations about Jacobi elliptic functions can be found in Refs. [10-12]. In an earlier paper [6], we proposed a unified algebraic method, called later on the mapping method [7-9], to obtain the exact periodic wave solutions in terms of the Jacobi elliptic functions for a large variety of nonlinear PDEs. Under limit conditions, more solitary wave, kink wave (or shock wave), and trigonometric periodic wave solutions may be obtained. In this paper, we will use a new algebraic method, the modified mapping method, to obtain more exact periodic wave solutions for some nonlinear PDEs. The basic idea of the method is as follows. For a given nonlinear evolution equation, say, in three variables

$$
N\left(u, u_{t}, u_{x}, u_{y}, u_{x x}, \cdots\right)=0,
$$

we seek for its travelling wave solution of the form

$$
u(x, t) \equiv u(\xi), \quad \xi=k x+l y-\omega t .
$$

The substitution of Eq. (5) into Eq. (4) yields an ordinary differential equation of $u(\xi)$. Then $u(\xi)$ is expanded into a polynomial in $f(\xi)$

$$
u(\xi)=\sum_{i=0}^{n} A_{i} f^{i}+\sum_{i=1}^{n} B_{i} f^{-i},
$$

where $A_{i}$ and $B_{i}$ are constants to be determined, $n$ is fixed by balancing the linear term of the highest order with a nonlinear term in Eq. (4), and $f$ satisfies the following equation (the first kind of elliptic equation):

$$
f^{\prime \prime}=p f+q f^{3}, \quad f^{\prime 2}=p f^{2}+\frac{1}{2} q f^{4}+r,
$$

where $p, q$, and $r$ are constants to be determined. After Eq. (6) with Eq. (7) is substituted into the ordinary differential equation, the coefficients $A_{i}, B_{i}, k, l, \omega, p, q$, and $r$ may be determined. If any of the parameters is left unspecified, it is regarded as being arbitrary for the solution to Eq. (4). Thus Eq. (6) establishes a new algebraic mapping relation between the solution to Eq. (7) and that of Eq. (4). Due to the entrance of three parameters $p, q$, and $r$, Eq. (7) has a rich structure of solutions, which will be seen in the study of the system in Sec. 2. When $B_{i}=0$, this method is named the mapping method [6-9]. In this paper, we take the Nizhnik-Novikov-Veselov (NNV) equation as an example to illustrate our approach and abundant periodic wave solutions are obtained. Limit cases are studied and some new solitary wave solutions and trigonometric periodic wave solutions are also found. 


\section{Exact periodic wave solutions to the NNV equation}

The (2+1)-dimensional Nizhnik-Novikov-Veselov equation [13, 14]

$$
u_{t}=a u_{x x x}+b u_{y y y}-3 a(u v)_{x}-3 b(u w)_{y}, \quad u_{x}=v_{y}, \quad u_{y}=w_{x},
$$

is the only known isotropic Lax integrable extension of the well-known (1+1)-dimensional KdV equation. Boiti et al. [15] solved the NNV equation via IST. Tagami [16] obtained the soliton-like solutions of the NNV equation by means of the Bäcklund transformation. The physical importance of Eq. (8) can be easily found in Refs. $[13,14]$. However, the NNV equation may possess many interesting solution structures that have not yet been found. In what follows, we will obtain the exact periodic wave solutions to the NNV equation by using the modified mapping method. Substituting Eq. (5) into Eq. (8) and integrating once, we obtain

$$
\begin{aligned}
& \left(a k^{3}+b l^{3}\right) u^{\prime \prime}+\omega u-3 a k u v-3 b l u w=C_{1}, \\
& k u=l v+C_{2}, \quad l u=k w+C_{3},
\end{aligned}
$$

where $C_{i}$ are integration constants, and from which we get

$$
A u^{\prime \prime}-B u^{2}+C u=D
$$

with

$$
\begin{aligned}
& A=k l\left(a k^{3}+b l^{3}\right), \quad B=3\left(a k^{3}+b l^{3}\right), \\
& C=k l \omega+3 a k^{2} C_{2}+3 b l^{2} C_{3}, \quad D=k l C_{1} .
\end{aligned}
$$

According to the method described above, we assume that Eq. (10) has the solution of the form

$$
u=A_{0}+A_{1} f+A_{2} f^{2}+B_{1} f^{-1}+B_{2} f^{-2},
$$

where $A_{i}$ and $B_{i}$ are constants to be determined and $f$ satisfies Eq. (7). The substitution of Eq. (12) into Eq. (10) and the use of Eq. (7) yield (equating the coefficients of the same powers of $f$ )

$$
\begin{aligned}
& 3 q A A_{2}-B A_{2}^{2}=0, \quad q A A_{1}-2 B A_{1} A_{2}=0, \\
& 4 p A A_{2}-B\left(A_{1}^{2}+2 A_{0} A_{2}\right)+C A_{2}=0, \\
& p A A_{1}-B\left(2 A_{0} A_{1}+2 A_{2} B_{1}\right)+C A_{1}=0, \\
& A\left(q B_{2}+2 r A_{2}\right)-B\left(A_{0}^{2}+2 A_{1} B_{1}+2 A_{2} B_{2}\right)+C A_{0}=D, \\
& p A B_{1}-B\left(2 A_{0} B_{1}+2 A_{1} B_{2}\right)+C B_{1}=0, \\
& 4 p A B_{2}-B\left(B_{1}^{2}+2 A_{0} B_{2}\right)+C B_{2}=0, \\
& 2 r A B_{1}-2 B B_{1} B_{2}=0, \quad 6 r A B_{2}-B B_{2}^{2}=0,
\end{aligned}
$$

from which we obtain three sets of the solutions 


$$
A_{0}=\frac{4 p A+C}{2 B}, \quad A_{2}=\frac{3 q A}{B}, \quad A_{1}=B_{1}=B_{2}=0
$$

and $A_{0}, A_{1}, A_{2}, B_{1}$ are the same as Eq. (14), but

$$
B_{2}=\frac{6 r A}{B}
$$

and

$$
A_{0}=\frac{4 p A+C}{2 B}, \quad B_{2}=\frac{6 r A}{B}, \quad A_{1}=A_{2}=B_{1}=0 .
$$

Because of the properties of the Jacobi elliptic functions, Eqs. (14) and (16) result in the same solution and we omit Eq. (16). Hence we get the two sets of the exact solutions to Eq. (8) as follows

$$
u_{1}=\frac{2}{3} p k l+\frac{k l \omega+3 a k^{2} C_{2}+3 b l^{2} C_{3}}{6\left(a k^{3}+b l^{3}\right)}+q k l f^{2}(\xi)
$$

and

$$
u_{2}=\frac{2}{3} p k l+\frac{k l \omega+3 a k^{2} C_{2}+3 b l^{2} C_{3}}{6\left(a k^{3}+b l^{3}\right)}+q k l f^{2}(\xi)+2 r k l f^{-2}(\xi),
$$

where we have used Eq. (11), $\xi=k x+l y-\omega t$ and $f$ satisfies Eq. (7). From the last one of Eq. (9) one can easily obtain the expressions of $v$ and $w$ and they are omitted throughout this paper. In what follows, we discuss the specific forms of the solutions Eqs. (17) and (18) according to the different values of the parameters $p, q$, and $r$.

Case 1. $p=-\left(1+m^{2}\right), \quad q=2 m^{2}, \quad r=1$.

Equation (7) has the solutions $f(\xi)=\operatorname{sn} \xi$ or $f(\xi)=\operatorname{cd} \xi \equiv \operatorname{cn} \xi / \operatorname{dn} \xi$. Thus, from Eqs. (17) and (18) we get the periodic wave solutions

$$
u_{1}=-\frac{2}{3}\left(1+m^{2}\right) k l+\frac{k l \omega+3 a k^{2} C_{2}+3 b l^{2} C_{3}}{6\left(a k^{3}+b l^{3}\right)}+2 m^{2} k l \operatorname{sn}^{2}(k x+l y-\omega t)
$$

or

$$
u_{1}=-\frac{2}{3}\left(1+m^{2}\right) k l+\frac{k l \omega+3 a k^{2} C_{2}+3 b l^{2} C_{3}}{6\left(a k^{3}+b l^{3}\right)}+2 m^{2} k l \mathrm{~cd}^{2}(k x+l y-\omega t)
$$

and

$$
\begin{aligned}
u_{2}= & -\frac{2}{3}\left(1+m^{2}\right) k l+\frac{k l \omega+3 a k^{2} C_{2}+3 b l^{2} C_{3}}{6\left(a k^{3}+b l^{3}\right)}+2 m^{2} k l \mathrm{sn}^{2}(k x+l y-\omega t) \\
& +2 k \operatorname{lns}^{2}(k x+l y-\omega t)
\end{aligned}
$$

or

$$
\begin{aligned}
u_{2}= & -\frac{2}{3}\left(1+m^{2}\right) k l+\frac{k l \omega+3 a k^{2} C_{2}+3 b l^{2} C_{3}}{6\left(a k^{3}+b l^{3}\right)}+2 m^{2} k l c d^{2}(k x+l y-\omega t) \\
& +2 k l \mathrm{dc}^{2}(k x+l y-\omega t)
\end{aligned}
$$


where $\mathrm{ns} \xi \equiv 1 / \mathrm{sn} \xi$ and $\mathrm{dc} \xi \equiv 1 / \mathrm{cd} \xi$. As $m \rightarrow 0$, from Eqs. (21) and (22) one has the trigonometric periodic wave solutions

$$
u_{2}=-\frac{2}{3} k l+\frac{k l \omega+3 a k^{2} C_{2}+3 b l^{2} C_{3}}{6\left(a k^{3}+b l^{3}\right)}+2 k l \csc ^{2}(k x+l y-\omega t)
$$

or

$$
u_{2}=-\frac{2}{3} k l+\frac{k l \omega+3 a k^{2} C_{2}+3 b l^{2} C_{3}}{6\left(a k^{3}+b l^{3}\right)}+2 k l \sec ^{2}(k x+l y-\omega t) .
$$

As $m \rightarrow 1$, the corresponding solitary wave solutions read

$$
u_{1}=-\frac{4}{3} k l+\frac{k l \omega+3 a k^{2} C_{2}+3 b l^{2} C_{3}}{6\left(a k^{3}+b l^{3}\right)}+2 k l \tanh ^{2}(k x+l y-\omega t)
$$

or

$$
\begin{aligned}
u_{2}= & -\frac{4}{3} k l+\frac{k l \omega+3 a k^{2} C_{2}+3 b l^{2} C_{3}}{6\left(a k^{3}+b l^{3}\right)}+2 k l \tanh ^{2}(k x+l y-\omega t) \\
& +2 k l \operatorname{coth}^{2}(k x+l y-\omega t)
\end{aligned}
$$

Case 2. $p=2 m^{2}-1, q=-2 m^{2}, r=m^{2} \equiv 1-m^{2}$

From Eq. (7) we have $f(\xi)=\operatorname{cn} \xi$, and Eq. (8) has the periodic wave solutions

$$
u_{1}=\frac{2}{3}\left(2 m^{2}-1\right) k l+\frac{k l \omega+3 a k^{2} C_{2}+3 b l^{2} C_{3}}{6\left(a k^{3}+b l^{3}\right)}-2 m^{2} k l \mathrm{cn}^{2}(k x+l y-\omega t)
$$

and

$$
\begin{aligned}
u_{2}= & \frac{2}{3}\left(2 m^{2}-1\right) k l+\frac{k l \omega+3 a k^{2} C_{2}+3 b l^{2} C_{3}}{6\left(a k^{3}+b l^{3}\right)}-2 m^{2} k l \operatorname{cn}^{2}(k x+l y-\omega t) \\
& +2\left(1-m^{2}\right) k l n c^{2}(k x+l y-\omega t),
\end{aligned}
$$

where nc $\xi \equiv 1 / \mathrm{cn} \xi$. As $m \rightarrow 0$, Eq. (28) degenerates to Eq. (24), and as $m \rightarrow 1$, Eqs. (27) and (28) degenerate to Eq. (25).

Case 3. $p=2-m^{2}, \quad q=-2, \quad r=-\left(1-m^{2}\right)$.

The solution of $\mathrm{Eq} .(7)$ reads $f(\xi)=\operatorname{dn} \xi$. So we get the periodic wave solutions of Eq. (8)

$$
u_{1}=\frac{2}{3}\left(2-m^{2}\right) k l+\frac{k l \omega+3 a k^{2} C_{2}+3 b l^{2} C_{3}}{6\left(a k^{3}+b l^{3}\right)}-2 k l \mathrm{dn}^{2}(k x+l y-\omega t)
$$

and

$$
\begin{aligned}
u_{2}= & \frac{2}{3}\left(2-m^{2}\right) k l+\frac{k l \omega+3 a k^{2} C_{2}+3 b l^{2} C_{3}}{6\left(a k^{3}+b l^{3}\right)}-2 k l \mathrm{dn}^{2}(k x+l y-\omega t) \\
& -2\left(1-m^{2}\right) k l n d^{2}(k x+l y-\omega t),
\end{aligned}
$$

where $\mathrm{nd} \xi \equiv 1 / \operatorname{dn} \xi$. As $m \rightarrow 1$, Eqs. (29) and (30) degenerate to Eq. (25). 
Case 4. $p=-\left(1+m^{2}\right), q=2, r=m^{2}$.

We have $f(\xi)=\mathrm{ns} \xi$ or $f(\xi)=\mathrm{d} c \xi$. From Eq. (17) one gets

$$
u_{1}=-\frac{2}{3}\left(1+m^{2}\right) k l+\frac{k l \omega+3 a k^{2} C_{2}+3 b l^{2} C_{3}}{6\left(a k^{3}+b l^{3}\right)}+2 k l \operatorname{ns}^{2}(k x+l y-\omega t)
$$

or

$$
u_{1}=-\frac{2}{3}\left(1+m^{2}\right) k l+\frac{k l \omega+3 a k^{2} C_{2}+3 b l^{2} C_{3}}{6\left(a k^{3}+b l^{3}\right)}+2 k l \mathrm{dc}^{2}(k x+l y-\omega t),
$$

and from Eq. (18) one obtains Eqs. (21) and (22) again. As $m \rightarrow$ 0, Eqs. (31) and (32) degenerate to Eqs. (23) and (24), respectively. As $m \rightarrow 1$, Eq. (31) degenerates to

$$
u_{1}=-\frac{4}{3} k l+\frac{k l \omega+3 a k^{2} C_{2}+3 b l^{2} C_{3}}{6\left(a k^{3}+b l^{3}\right)}+2 k l \operatorname{coth}^{2}(k x+l y-\omega t)
$$

Case 5. $p=2-m^{2}, q=2, r=1-m^{2}$.

Equation (7) has the solution $f(\xi)=\operatorname{cs} \xi \equiv \operatorname{cn} \xi / \operatorname{sn} \xi$. And the periodic wave solutions of Eq. (8) are

$$
u_{1}=\frac{2}{3}\left(2-m^{2}\right) k l+\frac{k l \omega+3 a k^{2} C_{2}+3 b l^{2} C_{3}}{6\left(a k^{3}+b l^{3}\right)}+2 k l \operatorname{cs}^{2}(k x+l y-\omega t)
$$

and

$$
\begin{aligned}
u_{2}= & \frac{2}{3}\left(2-m^{2}\right) k l+\frac{k l \omega+3 a k^{2} C_{2}+3 b l^{2} C_{3}}{6\left(a k^{3}+b l^{3}\right)}+2 k l \operatorname{cs}^{2}(k x+l y-\omega t) \\
& +2\left(1-m^{2}\right) k l \operatorname{sc}^{2}(k x+l y-\omega t),
\end{aligned}
$$

where $\operatorname{sc} \xi \equiv 1 / \operatorname{cs} \xi$. As $m \rightarrow 0$, Eqs. (34) and (35) degenerate to Eq. (23) and

$$
\begin{aligned}
u_{2}= & \frac{4}{3} k l+\frac{k l \omega+3 a k^{2} C_{2}+3 b l^{2} C_{3}}{6\left(a k^{3}+b l^{3}\right)}+2 k l \cot ^{2}(k x+l y-\omega t) \\
& +2 k l \tan ^{2}(k x+l y-\omega t),
\end{aligned}
$$

respectively. As $m \rightarrow 1$, Eqs. (34) and (35) degenerate to Eq. (33)

Case 6. $p=2 m^{2}-1, q=2, r=-m^{2}\left(1-m^{2}\right)$.

We have $f(\xi)=\mathrm{ds} \xi \equiv \operatorname{dn} \xi / \operatorname{sn} \xi$. Thus we get the periodic wave solutions to Eq. (8)

$$
u_{1}=\frac{2}{3}\left(2 m^{2}-1\right) k l+\frac{k l \omega+3 a k^{2} C_{2}+3 b l^{2} C_{3}}{6\left(a k^{3}+b l^{3}\right)}+2 k l \mathrm{ds}^{2}(k x+l y-\omega t)
$$

and

$$
\begin{aligned}
u_{2}= & \frac{2}{3}\left(2 m^{2}-1\right) k l+\frac{k l \omega+3 a k^{2} C_{2}+3 b l^{2} C_{3}}{6\left(a k^{3}+b l^{3}\right)}+2 k l \mathrm{ds}^{2}(k x+l y-\omega t) \\
& -2 m^{2}\left(1-m^{2}\right) k l_{\mathrm{sd}}{ }^{2}(k x+l y-\omega t),
\end{aligned}
$$


where $\mathrm{sd} \xi \equiv 1 / \mathrm{ds} \xi$. As $m \rightarrow 0$, Eqs. (37) and (38) degenerate to Eq. (23), and as $m \rightarrow 1$, Eqs. (37) and (38) degenerate to Eq. (33).

Case 7. $p=2 m^{2}-1, q=2\left(1-m^{2}\right), r=-m^{2}$.

Equation (7) has the solution $f(\xi)=n c \xi \equiv 1 / \operatorname{cn} \xi$. So the periodic wave solutions of Eq. (8) read

$$
\begin{aligned}
u_{1}= & \frac{2}{3}\left(2 m^{2}-1\right) k l+\frac{k l \omega+3 a k^{2} C_{2}+3 b l^{2} C_{3}}{6\left(a k^{3}+b l^{3}\right)} \\
& +2\left(1-m^{2}\right) k l n c^{2}(k x+l y-\omega t)
\end{aligned}
$$

and Eq. (28). As $m \rightarrow$ 0, Eq. (39) degenerates to Eq. (24).

Case 8. $p=2-m^{2}, q=2\left(1-m^{2}\right), r=1$.

The solution of Eq. (7) reads $f(\xi)=\operatorname{sc} \xi \equiv \operatorname{sn} \xi / \mathrm{cn} \xi$. The periodic wave solutions of Eq. (8) are

$$
\begin{aligned}
u_{1}= & \frac{2}{3}\left(2-m^{2}\right) k l+\frac{k l \omega+3 a k^{2} C_{2}+3 b l^{2} C_{3}}{6\left(a k^{3}+b l^{3}\right)} \\
& +2\left(1-m^{2}\right) k l_{s c}^{2}(k x+l y-\omega t)
\end{aligned}
$$

and Eq. (35). As $m \rightarrow$ 0, Eq. (40) degenerates to Eq. (24).

Case 9. $p=2 m^{2}-1, q=-2 m^{2}\left(1-m^{2}\right), r=1$.

We get $f(\xi)=\operatorname{sd} \xi \equiv \operatorname{sn} \xi / \operatorname{dn} \xi$. So we have

$$
\begin{aligned}
u_{1}= & \frac{2}{3}\left(2 m^{2}-1\right) k l+\frac{k l \omega+3 a k^{2} C_{2}+3 b l^{2} C_{3}}{6\left(a k^{3}+b l^{3}\right)} \\
& -2 m^{2}\left(1-m^{2}\right) k l s_{s}^{2}(k x+l y-\omega t)
\end{aligned}
$$

and Eq. (38).

Case 10. $p=2-m^{2}, q=-2\left(1-m^{2}\right), r=-1$.

From Eq. (7) one obtains $f(\xi)=\mathrm{nd} \xi$. Thus the periodic wave solutions to Eq. (8) read

$$
\begin{aligned}
u_{1}= & \frac{2}{3}\left(2-m^{2}\right) k l+\frac{k l \omega+3 a k^{2} C_{2}+3 b l^{2} C_{3}}{6\left(a k^{3}+b l^{3}\right)} \\
& -2\left(1-m^{2}\right) k \operatorname{lnd}^{2}(k x+l y-\omega t)
\end{aligned}
$$

and Eq. (30). 


\section{Conclusion and discussion}

New exact solutions to the NNV equation are studied by means of the modified mapping method. Exact periodic wave solutions in terms of the Jacobi elliptic functions are obtained. Limit cases are studied and new solitary wave solutions and trigonometric periodic wave solutions are found. It can be easily seen that the modified mapping method is applicable to a large variety of nonlinear PDEs, as long as even- and odd-order derivative terms do not coexist in the equation under consideration. By using the mapping method [6-9], we can only obtain the solution (17) to the NNV equation (8). However, by means of the modified mapping method, we can obtain not only the solution (17), but also the solution (18). The additional set of particular solution (18) develops singularity, generally speaking, at a finite point, i.e. for any fixed $t=t_{0}$, there exists $x_{0}$ at which the solution blows up. There is much current interest in the formation of so-called hot spots or blow up of solutions [17-20]. It appears that these singular solutions obtained in this paper will model these physical phenomena.

\section{References}

[1] E.J. Parkes, B.R. Duffy, P.C. Abbott, Phys. Lett. A 295, 280 (2002).

[2] E.G. Fan, B.Y.C. Hon, Phys. Lett. A 292, 335 (2002).

[3] S.D. Liu, Z.T. Fu, S.K. Liu, Q. Zhao, Commun. Theor. Phys. 39, 167 (2003).

[4] H.T. Chen, H.Q. Zhang, Chaos, Solitons and Fractals 15, 585 (2003).

[5] Z.Y. Yan, J. Phys. A 36, 1961 (2003).

[6] Y.Z. Peng, Acta Phys. Pol. A 103, 417 (2003).

[7] Y.Z. Peng, Phys. Lett. A 314, 401 (2003).

[8] Y.Z. Peng, J. Phys. Soc. Jpn. 72, 1356 (2003).

[9] Y.Z. Peng, Chin. J. Phys. 41, 103 (2003).

[10] F. Bowman, Introduction to Elliptic Functions with Applications, Universities, London 1959.

[11] S.K. Liu, S.D. Liu, Nonlinear Equations in Physics, Peking University Press, Beijing 2000 (in Chinese).

[12] V. Prasolov, Y. Solovyev, Elliptic Functions and Elliptic Integrals, American Mathematical Society, Providence 1997.

[13] L.P. Nizhnik, Sov. Phys. Dokl. 25, 706 (1980).

[14] S.P. Novikov, A.P. Veselov, Physica D 18, 267 (1986).

[15] M. Boiti, J.J.P. Leon, M. Manna, F. Pempinelli, Inverse Probl. 2, 271 (1986).

[16] Y. Tagami, Phys. Lett. A 141, 116 (1989).

[17] N.A. Kudryashov, D. Zargaryan, J. Phys. A 29, 8067 (1996).

[18] C.J. Coleman, J. Aust. Math. Soc. Ser. B 33, 1 (1992).

[19] N.F. Smyth, J. Aust. Math. Soc. Ser. B 33, 403 (1992).

[20] P.A. Clarkson, E.L. Mansfield, Physica D 70, 250 (1993). 